\title{
Oncolytic Viruses and Immune Checkpoint Inhibitors
}

\author{
Jean-Francois Fonteneau ${ }^{1,2^{*}}$ \\ ${ }^{1}$ INSERM, UMR892, Institut de Recherche en Santé de l'Université de Nantes, France \\ ${ }^{2}$ CNRS, UMR6299, Institut de Recherche en Santé de l'Université de Nantes, France
}

*Corresponding author: Jean-Francois Fonteneau, INSERM U892, Institut de Recherche Thérapeutique de l'Université de Nantes, 8 quai Moncousu, BP 70721,44007 Nantes, France, Tel: 33-228080239; Fax: 33-228080204; E-mail: jean-francois.fonteneau@inserm.fr

Received date: November 11, 2016, Accepted date: November 17, 2016, Published date: November 25, 2016

Copyright: (c) 2016 Fonteneau JF. This is an open-access article distributed under the terms of the Creative Commons Attribution License, which permits unrestricted use, distribution, and reproduction in any medium, provided the original author and source are credited.

\section{Editorial}

Oncolytic immunotherapy consists in the use of non-pathogenic replicative viruses that infect preferentially or exclusively tumor cells and induces immunogenic cell death able to induce or stimulate an antitumor immune response [1]. In October 2015, a first oncolytic virus $(\mathrm{OV})$ was approved by the FDA for the treatment of metastatic melanoma: the Talimogene Laherparepvec (T-Vec) from Amgen that is now commercialized under the name of Imlygic. T-Vec is a modified type I herpes simplex virus (HSV-I) with insertion and deletion of two genes. The two inserted genes encode the human granulocytemacrophage colony-stimulating factor (GM-CSF) to stimulate the antitumor immune response and the HSV US11 protein to increase viral replication. The two deleted genes encode two virulence factors: ICP47 that normally inhibits the transport of peptide from cytoplasm to endoplasmic reticulum for HLA class I presentation in infected cell and ICP34.5 a neurovirulence factor.

$\mathrm{T}$-Vec was assessing in a multicentric phase III clinical trial to treat metastatic melanoma by intratumoral injections [2]. This OV was compared with GM-CSF for the treatment of 436 patients randomly assigned in two arms: patients receiving T-Vec vs. patients receiving GM-CSF. T-Vec was safe for the patients with low grade side effects and resulted in a higher durable responses rate and longer mean overall survival. It is interesting to note that the clinical benefits were better in early stages of metastatic melanoma (IIIb, IIIc and IVM1a) compared to more advanced stages (IVM1b and IVM1c) and in patients receiving the $\mathrm{T}-\mathrm{Vec}$ as a first line treatment compared to the TVec administered as second line. In contrary, no difference of efficacy was observed in patients that were seropositive to HSV1, compared to seronegative ones.

Recently, this work was completed by a characterization of the clinical response induced by T-Vec at the level of individual lesions [3]. This study shows that the size of $64 \%$ of T-Vec injected lesion diminish more than $50 \%$. Interestingly, a reduction of size superior to $50 \%$ was also observed in $34 \%$ of non-injected non-visceral lesions and in $15 \%$ of visceral lesions. These results prove that $\mathrm{T}-\mathrm{Vec}$ has induced an antitumor immune response that is able to diminish or eradicate distant lesions.

Given that $\mathrm{T}-\mathrm{Vec}$ is able to induce an antitumor immune response in patients, several phase I clinical trials have started to evaluate the combination of this OV with immune checkpoint inhibitor (ICI): pembrolizumab (anti-PD-1) for treatment of unresectable stage IIIB to IVM1c melanoma (NCT02263508) and for recurrent metastatic squamous cell carcinoma of the head and neck (NCT02626000) or ipilimumab (anti-CTLA-4) for unresectable stage IIIB to IVM1c melanoma (NCT01740297). Since 2011, ICI revolutionized immunotherapy of cancer by blocking molecules that are expressed inside tumors and that block the antitumor immune response, especially cytotoxic functions of T lymphocytes [4]. In August 2016, a first report was published regarding the use of a combination of T-Vec with ipilimumab in a phase $\mathrm{Ib}$ clinical trial for the treatment of metastatic melanoma [5]. The authors show no additional adverse effects with this combination compared to what has been observed when each drug is used alone. Furthermore, their results suggest that the combination of both drugs is more efficient than each drug used alone. No result is published yet on the combination of T-Vec with pembrolizumab, but encouraging preliminary results were presented by Dr Robert Andtbacka at the "10th International Oncolytic Virus Meeting", in Vancouver, Canada, in October 2016 (abstract \#10).

Other OV are evaluated in phase III clinical trials, such as JX594, a modified vaccinia virus in combination with sorafenib for the treatment of advanced hepatocellular carcinoma (NCT02562755), CG0070, a modified adenovirus for the treatment of BCG therapy resistant non muscle invasive bladder (NCT02365818), Reolysin, a Reovirus Serotype 3 Dearing in combination with paclitaxel and carboplatin for the treatment of Squamous Cell Carcinoma of the Head and Neck (NCT01166542) or Toca511, a modified gamma retrovirus and TocaFC (5-fluorocytosine) for the treatment of multiform glioblastoma after surgical resection (NCT02414165) [6-8]. It is tempting to speculate that they will be evaluated in combination with ICI in the near future. Indeed, oncolytic viruses by inducing immunogenic cell death and type I IFN response in the tumor bed can induce infiltration by immune cells, notably cytotoxic $\mathrm{T}$ cells and natural killer cells that will be potentiated by ICI. Indeed, in all published preclinical model, there is an additive or synergic effects of OV with ICI [9].

\section{References}

1. Lichty BD, Breitbach CJ, Stojdl DF, Bell JC (2014) Going viral with cancer immunotherapy. Nat Rev Cancer 14: 559-567.

2. Andtbacka RHI, Kaufman HL, Collichio F, Amatruda T, Senzer N, et al. (2015) Talimogene Laherparepvec Improves Durable Response Rate in Patients With Advanced Melanoma. J Clin Oncol.

3. Andtbacka RH, Ross M, Puzanov I, Milhem M, Collichio F, et al. (2016) Patterns of Clinical Response with Talimogene Laherparepvec (T-VEC) in Patients with Melanoma Treated in the OPTiM Phase III Clinical Trial. Ann Surg Oncol 23: 4169-4177.

4. Kreamer KM (2014) Immune Checkpoint Blockade: A New Paradigm in Treating Advanced Cancer. J Adv Pract Oncol 5: 418-431.

5. Puzanov I, Milhem MM, Minor D, Hamid O, Li A, et al. (2016) Talimogene Laherparepvec in Combination With Ipilimumab in Previously Untreated, Unresectable Stage IIIB-IV Melanoma. J Clin Oncol 34: 2619-2626.

6. Pol J, Bloy N, Obrist F, Eggermont A, Galon J, et al. (2014) Trial Watch:: Oncolytic viruses for cancer therapy. Oncoimmunology 3: e28694. 
Citation: Fonteneau JF (2016) Oncolytic Viruses and Immune Checkpoint Inhibitors. Immunother Open Acc 2: e105. doi: $10.4172 / 2471-9552.1000 \mathrm{e} 105$

Page 2 of 2

7. Pol J (2016) Trial Watch-Oncolytic viruses and cancer therapy Oncoimmunology 5: e1117740.

8. Vacchelli E, Eggermont A, Sautès-Fridman C, Galon J, Zitvogel L, et al. (2013) Trial watch: Oncolytic viruses for cancer therapy. Oncoimmunology 2: e24612.
9. Marchini A, EM Scott, Rommelaere J (2016) Overcoming Barriers in Oncolytic Virotherapy with HDAC Inhibitors and Immune Checkpoint Blockade. Viruses 8: 9. 